\title{
Oriented Attachment is a Major Control Mechanism to Form Nail-like Mn-doped ZnO Nanocrystals
}

\author{
Samuel Patterson ${ }^{1}$, Priyanka Arora ${ }^{\dagger 1}$, Paige Price ${ }^{1}$, Jasper W. Dittmar ${ }^{1}$, Vijay Kumar Das ${ }^{1}$, \\ Maren Pink ${ }^{1}$, Barry Stein ${ }^{2}$, David Gene Morgan ${ }^{1}$, Yaroslav Losovyj ${ }^{1}$, Kallum M. Koczkur ${ }^{1}$, Sara \\ E. Skrabalak ${ }^{1}$ Lyudmila M. Bronstein $* 1,3,4$ \\ ${ }^{1}$ Indiana University, Department of Chemistry, Bloomington, IN 47405, USA \\ ${ }^{2}$ Indiana University, Department of Biology, Bloomington, IN 47405, USA
}

${ }^{3}$ A.N. Nesmeyanov Institute of Organoelement Compounds, Russian Academy of Sciences, 28 Vavilov St., Moscow, 119991 Russia

${ }^{4}$ King Abdulaziz University, Faculty of Science, Department of Physics, Jeddah, Saudi Arabia

\begin{abstract}
Here, we present a controlled synthesis of Mn-doped ZnO nanoparticles (NPs) with predominantly nail-like shapes, whose formation occurs via tip-to-base oriented attachment of initially formed nanopyramids, followed by levelling of sharp edges that lead to smooth singlecrystalline "nails". This shape is prevalent in non-coordinating solvents such as octadecene and octadecane. Yet, the double bond in the former promotes oriented attachment. In contrast, Mndoped ZnO NP synthesis in a weakly coordinating solvent, benzyl ether, results in dendritic
\end{abstract}


structures due to random attachment of initial NPs. Mn-doped ZnO NPs possess a hexagonal wurtzite structure, and in the majority of cases, the NP surface is enriched with Mn, indicating a migration of $\mathrm{Mn}^{2+}$ ions to the NP surface during the NP formation. When the NP formation is carried out without the addition of octadecyl alcohol, which serves as surfactant and reaction initiator, large, concave pyramid dimers are formed whose attachment takes place via basal planes. UV-Vis and photoluminescence spectra of these NPs confirm the utility of controlling NP shape in order to tune electro-optical properties.

Key words: Mn-doped $\mathrm{ZnO}$, nanoparticles, shape, oriented attachment

\section{Introduction}

The Darwinian concept "Function follows form" is well known in biology. ${ }^{1-2}$ More recently, this concept was introduced in materials science when exploring two-dimensional supramolecular assembly at surfaces ${ }^{3}$ and demonstrating that solar cell performance is strongly dependent on donor polymer side chain identity. ${ }^{4}$ The influence of form (size and shape) on function (properties) is especially pronounced for nanoparticles (NPs) as many properties (catalytic, optical, magnetic, stability) are size and shape dependent at the nanoscale. These dependencies are well documented for metal ${ }^{5-6}$ and metal compound ${ }^{7}$ NPs as well as for metal oxide NPs. ${ }^{8-9}$

A great deal of attention has been paid to semiconductor nanoparticles due to their interesting optical, electromagnetic, and electro/photocatalytic properties. The controlled modification of nanoparticle size and shape is important for applications in photocatalysis, green energy, biosensing, and diagnostics. ${ }^{10-14} \mathrm{ZnO}$ nanoparticles attracted considerable attention because they are inexpensive, possess low toxicity and can assume a wide variety of properties based on control 
of their size, shape, and composition. They can be synthesized by precipitation with a base, ${ }^{9,15}$ by solvothermal and sol-gel methods, ${ }^{16-19}$ etc.

Doping is a common technique to modify nanoparticle shape and properties. Previous work has shown that doping of $\mathrm{ZnO} \mathrm{NPs}$ with $\mathrm{Ni}, \mathrm{Cu}, \mathrm{Fe}, \mathrm{Mn}, \mathrm{V}$, and $\mathrm{Co}$ ions allows one to control ferromagnetic, catalytic, optical, and other properties. ${ }^{16,20-24}$ Copper doping in ZnO NPs has allowed for increased voltage generation, storage capacity, and energy conversion efficiency in photoelectrochemical cells. ${ }^{21} \mathrm{Ni}$ doping influenced structural, optical and photocatalytic properties of $\mathrm{ZnO}^{22} \mathrm{Mg}$ doping, depending on the molar ratio of a dopant precursor in a solvothermal process, yields well-defined nanocrystals with shapes ranging from tetrapods to ultrathin nanowires. Moreover, these NPs exhibit tunable optoelectronic properties. ${ }^{16}$ For Mn doping, the shape of doped $\mathrm{ZnO}$ NPs was modulated from three dimensional tetrapods to zero-dimensional spherical nanoparticles in a single reaction scheme by changing the reaction time. ${ }^{25}$ The authors suggested a growth mechanism of doped $\mathrm{ZnO}$ NPs, according to which tetrapod arms break off with passage of time due to crystal strain, with the arms smoothing over time leading to formation of spheres. ${ }^{25}$ Optical absorption tuning of Mn-doped ZnO NPs was also demonstrated by varying Mn doping levels. This fascinating work stimulated our own exploration of shape control in the syntheses of Mn-doped ZnO NPs.

Here, we focused on exploring the mechanism of Mn-doped ZnO NP formation and discovered that nail-like NPs are formed due to tip-to-base oriented attachment of initial pyramid-shaped NPs. This process is greatly promoted in a non-coordinating solvent containing a double bond (octadecene) and completely impeded in a weakly coordinating solvent (benzyl ether). These findings pave the way for a better mechanistic understanding of how these types of NPs form. 


\section{Experimental part}

\section{Materials}

Zinc stearate $(\mathrm{Zn}(\mathrm{St}) 2$, purum, $10-12 \% \quad \mathrm{Zn}$ basis $)$, tetramethylammonium hydroxide pentahydrate, $\left(\mathrm{CH}_{3}\right)_{4} \mathrm{~N}(\mathrm{OH}) \times 5 \mathrm{H}_{2} \mathrm{O}(\mathrm{TMAH}, \geq 97 \%)$, 1-octadecene (ODE, $\left.\geq 95 \%\right)$, 1-octadecanol (ODA, 95\%), octadecane (99\%), stearic acid (HSt, 95\%), and benzyl ether (98\%) were purchased from Sigma-Aldrich and used as received. Manganese (II) stearate $\left(\mathrm{Mn}(\mathrm{St})_{2}, 99 \%\right)$ was purchased from American Elements and used without purification.

\section{Synthesis of Mn-doped ZnO NPs}

In a typical synthesis, $0.45 \mathrm{mmol}(0.2845 \mathrm{~g})$ of $\mathrm{Zn}(\mathrm{St})_{2}, 0.05 \mathrm{mmol}(0.0311 \mathrm{~g})$ of $\mathrm{Mn}(\mathrm{St}) 2$, and $10 \mathrm{~mL}$ of solvent were loaded into a $50 \mathrm{~mL}$ three-neck flask, then heated at $10{ }^{\circ} \mathrm{C} / \mathrm{min}$ under stirring to $300{ }^{\circ} \mathrm{C}$ under argon flow to yield a clear and colorless solution. The reaction chamber was opened briefly and $2.5 \mathrm{mmol}(0.676 \mathrm{~g})$ of ODA was added as a solid in the argon counter flow, causing the reaction temperature to drop to $\sim 290{ }^{\circ} \mathrm{C}$. The reaction flask was then resealed, reheated to $300{ }^{\circ} \mathrm{C}$, and kept at this temperature for the reaction duration before cooling to room temperature.

For hot addition reactions, $2.5 \mathrm{mmol}(0.676 \mathrm{~g})$ of ODA was heated to $200{ }^{\circ} \mathrm{C}$ in $5 \mathrm{~mL}$ of ODE under argon. Then the hot solution was transferred to the main reaction flask via a gas-tight syringe to initiate the NP formation.

NPs were precipitated by adding $9 \mathrm{~mL}$ of a 5:1 mixture of acetone:hexane to $\sim 1 \mathrm{~mL}$ of the reaction solution followed by centrifugation for $10 \mathrm{~min}$. The supernatant was removed, and the resulting pellet was washed twice with a 3:1 acetone:hexane mixture, followed by centrifugation and removal of the supernatant after each wash. The final pellet was dissolved in chloroform for further analysis. 
For analyzing probes, $0.5 \mathrm{~mL}$ aliquots were taken at time intervals from the reaction solution and precipitated immediately.

The key samples synthesized are listed in Table 1. MZO stands for Mn-doped ZnO.

Table 1. Conditions of syntheses of Mn-doped ZnO NPs. ${ }^{a}$

\begin{tabular}{|c|c|c|c|c|}
\hline $\begin{array}{l}\text { Sample } \\
\text { notation }\end{array}$ & $\begin{array}{l}\text { Reaction time, } \\
\text { min }\end{array}$ & Additive & Solvent & $\begin{array}{l}\text { Internal } \\
\text { Number }\end{array}$ \\
\hline MZO-1 & 10 & None & Octadecene & 61 \\
\hline MZO-2 & 60 & None & Octadecene & P-11 \\
\hline $\mathrm{MZO}-3^{\mathrm{b}}$ & 10 & None & Octadecene & 59 \\
\hline $\mathrm{MZO}-4^{\mathrm{b}}$ & 60 & None & Octadecene & P-4 \\
\hline MZO-5 & 10 & TMAH, 2 wt. $\%$ of $\mathrm{Mn}(\mathrm{St})_{2}$ & Octadecene & 51 \\
\hline MZO-6 & 10 & TMAH, 5 wt. $\%$ of $\mathrm{Mn}(\mathrm{St})_{2}$ & Octadecene & 47 \\
\hline MZO-7 & 10 & $\begin{array}{l}\text { TMA-HSt, } 2 \text { wt. } \% \text { of } \\
\operatorname{Mn}(\mathrm{St})_{2}\end{array}$ & Octadecene & 52 \\
\hline $\mathrm{MZO}-8^{\mathrm{c}}$ & 0 & None & Octadecene & 13 \\
\hline $\mathrm{MZO}-9^{\mathrm{c}}$ & 6 & None & Octadecene & 31 \\
\hline $\mathrm{MZO}-10^{\mathrm{c}}$ & 10 & None & Octadecene & 20 \\
\hline $\mathrm{MZO}-11^{\mathrm{c}}$ & 60 & None & Octadecene & 21 \\
\hline MZO-12 & 2 & None & Octadecene & 30 \\
\hline MZO-13 & 10 & None & Octadecane & 36 \\
\hline MZO-14 & 60 & None & Octadecane & 39 \\
\hline MZO-15 & 0 & None & Benzyl Ether & 79 \\
\hline MZO-16 & 10 & None & Benzyl Ether & 35 \\
\hline MZO-17 & 60 & None & Benzyl Ether & 42 \\
\hline MZO-18d & 100 & None & Octadecene & 69 \\
\hline MZO-19e & 100 & None & Octadecene & 75 \\
\hline
\end{tabular}




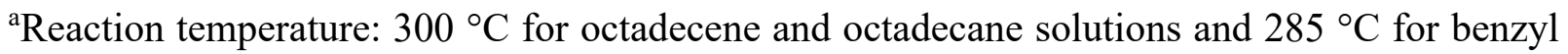
ether, ODA added as solid unless indicated otherwise; baddition of ODA in the $200{ }^{\circ} \mathrm{C}$ ODE solution; ${ }^{c}$ no stirring after the ODA addition; ${ }^{d}$ without ODA; ${ }^{e}$ probes taken in 20 and 40 min in the synthesis without ODA.

Synthesis of the TMAH and HSt product (TMA-St)

To simulate a potential impurity in $\mathrm{Mn}(\mathrm{St})_{2}, 1 \mathrm{mmol}$ of stearic acid was reacted with $1 \mathrm{mmol}$ of TMAOH in $10 \mathrm{~mL}$ of methanol at $50{ }^{\circ} \mathrm{C}$ for 30 minutes. The product (TMA-St) was separated via centrifugation and purified by washing with methanol three times followed by drying overnight in a vacuum oven.

\section{Characterization}

Transmission electron microscopy (TEM) images were acquired at an accelerating voltage of 80 $\mathrm{kV}$ on a JEOL JEM 1010 TEM and analyzed with the National Institute of Health developed image-package ImageJ to estimate NP diameters. Between 150 and 200 NPs were used for this analysis. For high-resolution TEM (HRTEM) and scanning TEM (STEM), a dispersed particle solution was drop-casted on a 300 mesh Cu grid (Ted-Pella, Formvar removed by dipping grid in chloroform). After 5 minutes, the grid was dipped in methanol, air dried for 30 minutes and then soaked in acetone overnight. Sample analysis by HRTEM and STEM were conducted on a JEOL JEM 3200FS TEM, equipped with a Gatan 4k x 4k UltraScan 4000 CCD camera, operating at 300 $\mathrm{kV}$ with a spot size of 1 . EDS spectra were obtained with an Oxford Instruments X-max ${ }^{\mathrm{N}} 100$ TLE silicon drift detector (SDD) interfaced to the JEOL JEM 3200FS TEM using the AZtec software package. Elemental mapping was conducted in the STEM-EDS mode.

Samples for scanning electron microscopy (SEM) were prepared by drop-casting a dispersed particle solution onto a cleaned Si wafer. After the solvent evaporated, the Si wafers were washed with methanol several times. Energy dispersive X-ray Spectroscopy (EDS) analysis was conducted 
on an Oxford Instruments X-Max $50 \mathrm{~mm}^{2}$ silicon drift detector (SDD) interfaced to the FEI Quanta SEM using the AZtec software package.

X-ray powder diffraction (XRD) patterns were collected on an Empyrean from PANalytical. Xrays were generated from a copper target with a wavelength $\mathrm{Cu} \mathrm{K} \alpha$ of $1.54187 \AA$ A. Soller slits, antiscatter slits, divergence slits and a nickel filter were in the beampath. During the measurement in reflection mode the sample was spinning with a revolution time of $4 \mathrm{~s}$. The measurements were performed with various step-sizes of and counting times. X-ray photoelectron spectroscopy (XPS) experiments were performed using PHI Versa Probe II instrument equipped with a monochromatic $\mathrm{Al} \mathrm{K} \alpha$ source. The X-ray power of $25 \mathrm{~W}$ at $15 \mathrm{kV}$ was used for a 200 micron beam size. XPS spectra with the energy step of $0.1 \mathrm{eV}$ were recorded using SmartSoft-XPS v2.0 and processed with PHI MultiPack v9.0 software. Peaks were fitted using Gaussians (G) and Lorentzians (L) line shapes and/or asymmetric shapes, i.e., a combination of Gaussians and Lorentzians with a 10-50\% of Lorentzian content. Shirley background was used for curve-fitting. NP samples for XPS were prepared by drop casting from chloroform solution onto the native surface of a $\mathrm{Si}(111)$ wafer.

UV-Vis spectra $(325-800 \mathrm{~nm})$ were collected on an Agilent Technologies Cary $60 \mathrm{UV}-\mathrm{Vis}$ spectrophotometer. Spectra were used to generate Tauc plots to determine optical band gaps.

Photoluminescence (PL) spectra were measured on a PTI QuantaMaster 300 steady state spectrofluorometer using a $75 \mathrm{~W}$ xenon laser as an excitation source. Samples dissolved in chloroform were diluted to $0.1 \mathrm{mg} / \mathrm{mL}$ in a stoppered quartz cuvette to prevent evaporation. A 325 $\mathrm{nm}$ excitation source was used and emission was measured in the range of 335-600 nm using a photomultiplier tube detector. Varying lamp intensity over different wavelengths was corrected for by using a reference diode detector. Each solution was measured at least three times to ensure reproducibility. 


\section{Results and Discussion}

\section{Shape and shape evolution in octadecene}

To study the influence of reaction parameters on the shape and shape evolution of Mn-doped ZnO NPs prepared by decomposition of Mn (10 mol. \%) and Zn stearates, we used a published procedure, ${ }^{25}$ in which the reaction began after the addition of ODA into the boiling ODE ( $\sim 300$ $\left.{ }^{\circ} \mathrm{C}\right)$ reaction solution. It is worth noting that here ODA serves as both surfactant and a reaction initiator via alcoholysis. ${ }^{16,25-26}$ We planned to follow a shape transformation from tetrapods to spheres as was discussed in ref. ${ }^{25}$ but chose to avoid a hazardous step, i.e., an injection of a hot $\left(200^{\circ} \mathrm{C}\right)$ ODA solution in ODE. Instead, ODA was added as a solid to the reaction solution, taking into consideration that ODA immediately dissolves at the reaction temperature. To our surprise, the sample obtained after the 10 min reaction time (timing began after the ODA addition) showed a few tetrapods, while the majority of the sample consisted of nail-like NPs. Some of these naillike NPs are indicated by red arrows in Figure 1a. Their length is about $30 \mathrm{~nm}$, while their width at the foot of the nail is about $6 \mathrm{~nm}$. It is noteworthy that similar shaped ZnO NPs called "towers" were observed by Peng and coauthors ${ }^{26}$ when reactions were carried out at the precursor concentrations $(0.4 \mathrm{mmol} / \mathrm{mL})$ an order of magnitude higher than their regular concentrations $(0.04$ $\mathrm{mmol} / \mathrm{mL})$ or the concentration used in our work $(0.05 \mathrm{mmol} / \mathrm{mL})$. In ref. ${ }^{26}$ the authors discussed that elongated structures are normally obtained at high precursor concentrations, but our work demonstrates that this condition is not necessarily a requirement.

The increase of the reaction time to 60 min made the "nails" smoother (Fig. 1b) and produced some faceted NPs observed in the $\mathrm{ZnO}$ synthesis, ${ }^{16}$ but no spherical particles. We assumed that the shape and shape evolution changes were caused by the addition of solid ODA, instead of the hot ODA solution. To eliminate this variance, the synthetic procedure was repeated with a hot 
injection of the ODA solution. However, the NP shapes and sizes were nearly the same as in the case of the solid ODA addition (Fig. S1, the Supporting Information, SI).
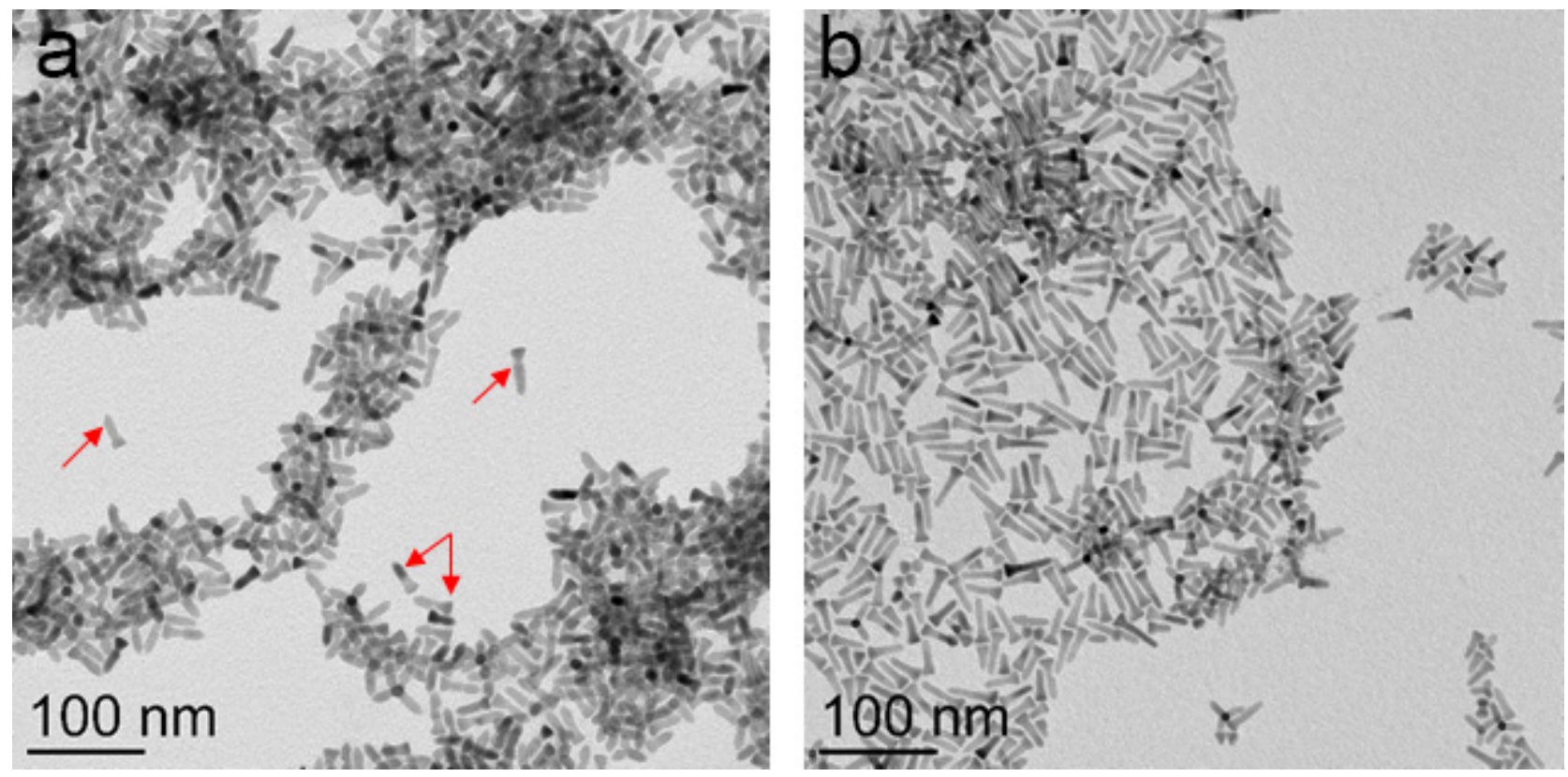

Figure 1. TEM images of MZO-1 and MZO-2 NPs obtained after 10 min (a) and 60 min (b), respectively, using solid ODA. Red arrows point to some nail-like NPs.

The question arises: What parameter could dramatically change not only the NP shape but also the shape evolution? Considering that the reaction conditions were fully reproduced from ref. ${ }^{25}$, the most likely cause of this phenomenon is a precursor purity/impurity. Ye and coauthors ${ }^{25}$ synthesized $\mathrm{Mn}(\mathrm{St})_{2}$ using a published procedure, washed it multiple times to remove $\mathrm{MnCl}_{2}$, TMAH and HSt, but never characterized the compound purity. They performed control experiments, in which they tested the presence of free HSt $(0.1 \mathrm{mmol}$ per $1 \mathrm{mmol}$ total of the Mn and Zn precursors) and of additional chloride ions on the NP formation. They concluded that the former slows down the reactivity of metal precursors, while the latter stimulates aggregation. However, neither addition of TMAH nor of the product of TMAH and HSt (TMA-St) was explored. Figure S2 (SI) shows that the addition of TMAH or TMA-St results in significant 
changes of the NP shapes. The addition of 2 wt.\% (towards $\left.\mathrm{Mn}(\mathrm{St})_{2}\right)$ of TMAH leads to much shorter nail-like particles (MZO-5), while with the increased amount of TMAH (MZO-6), mainly faceted particles are formed. In the case of the TMA-St (MZO-7), the NP shape change is even more dramatic as the faceted particles grow much larger and assemble into dimers and trimers. Despite the observed variations of the NP shapes, they did not emulate the results obtained by Ye and co-workers, ${ }^{25}$ thus, leaving the question open as to why particle shape and shape evolution are so different.

\section{Exploration of mechanism of nail-like NP formation}

To understand the mechanism of the nail-like NP formation, we identified the products of the reaction obtained immediately after the ODA addition and a few minutes after that. Especially revealing results were obtained when stirring was turned off after the ODA addition to slow the reaction down. Figure 2 demonstrates that at the very first moment after the ODA addition (MZO8), comparatively long structures are formed most likely by an assembly of four-five pyramid-like NPs as indicated by their jagged profile. Apparently these structures are unstable and already 6 min after the reaction began (MZO-9), they disassemble into individual pyramid-like NPs or their dimers (Fig. 2b). The sample obtained after 10 min (MZO-10) looks very similar (Fig. 2c), while after 60 min (MZO-11) the sample consists of some tetrapods and nail-like NPs (Fig. 2d), although the sharp features of individual pyramids are not yet smoothed out. 

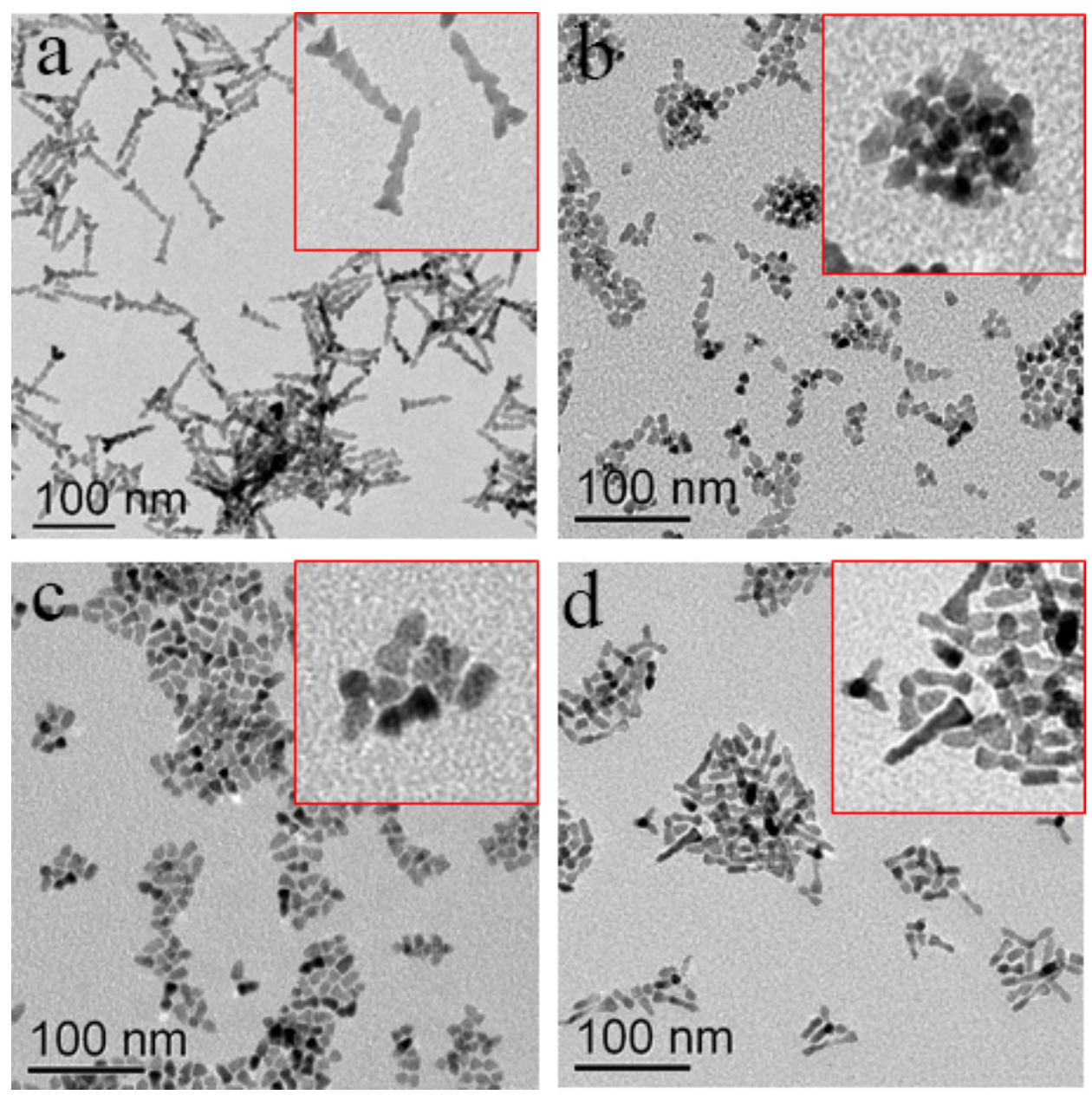

Figure 2. TEM images of NPs synthesized in octadecene for 0 (a, MZO-8), 6 (b, MZO-9), 10 (c, MZO-10), and $60 \mathrm{~min}(\mathrm{~d}, \mathrm{MZO}-11)$, with the stirring turned off after the ODA addition.

In analogous experiments but carried out with continuous stirring, the sample withdrawn after 2 min following the ODA addition (Fig. S3, SI, MZO-12), already contains smooth, nail-like NPs similar to those shown in Figure 1a. Only a few NPs (indicated by red arrows) show jagged profiles remaining after the attachment of pyramid-like NPs. Clearly, stirring accelerates the reaction to form nail-like NP. The influence of stirring on the NP size and shape has been discussed in the scientific literature for other metal oxides. ${ }^{27-28}$ In the absence of stirring, diffusion dominates the reaction, while mass transfer is limited. Stirring better promotes mass transfer in the reaction 
solution, thus, facilitating particle growth. The other important factor in the formation of nail-like NPs is flattening of sharp corners/edges of pyramid dimers and trimers resulting in a smooth surface shown in Figure 1b. This change in structure occurs because NP sharp features are energetically unfavorable and especially prone to dissolution at high reaction temperatures while formation of smooth surfaces energetically favorable. ${ }^{29}$

The above data indicate that the nail-like NPs are formed by the attachment of pyramid-like particles. But how does this attachment occur? To clarify the mechanism, we carried out HRTEM of two samples (Fig. 3): The NPs obtained after 10 min reaction time without stirring (MZO-10) showing the attachment of pyramids to each other (Fig. 2c) and the nail-like NPs obtained after 60 min of stirring (Fig. 1b, MZO-2) at otherwise identical conditions.

Fast Fourier Transform (FFT) patterns of the images demonstrate that both the pyramid dimers and nail-like NPs are single crystals, indicating that triangular pyramids, which are most likely formed immediately at the very first moment of the reaction, are prone to tip-to-base oriented attachment. ${ }^{30-33}$ As was discussed elsewhere, for a 1D oriented attachment, the electric dipole moment of the nanocrystals plays a determining role. ${ }^{34}$ Although at first these assembled pyramids are unstable and disintegrate in a few minutes (Fig. 2a and b), they are predisposed to further tipto-base attachment, followed by the formation of single crystalline nail-like NPs, whose sharp edges smooth out with passage of the reaction time. 

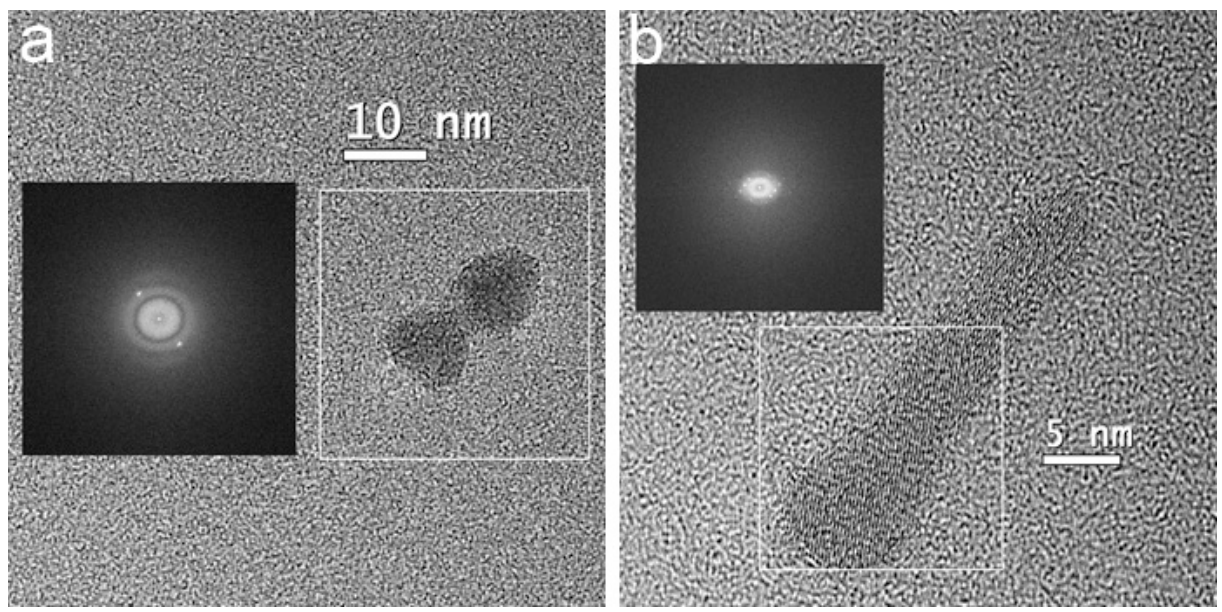

Figure 3. HRTEM images of NPs prepared in octadecene after $10 \mathrm{~min}$ without stirring (a, MZO10) and after 60 min with stirring (b, MZO-2). Insets show FFT patterns. White squares indicate areas chosen for the FFT analysis.

As is indicated in Figures S4 and S5 (SI), the nail-like NP growth can occur perpendicular to the (002) Miller plane $(0.261 \mathrm{~nm}$ interplanar distance), along the (100) plane $(0.286 \mathrm{~nm})$, and at an angle to the (101) plane $(0.249 \mathrm{~nm})$, depending on the basal plane, to which the tip of the other pyramid attaches to. The above distances are consistent with the interplanar distances reported for $\mathrm{ZnO}$ nanocrystals. ${ }^{26,35}$ If three or four pyramids are attached to each other without oriented attachment, tripods or tetrapods are formed. Considering that tetrapods are not dominant at our conditions, oriented attachment is favored.

\section{Solvent effect on nanoparticle shape}

The NP shape can be strongly influenced by solvent effects due to the presence of functional groups or the solvent polarity. ${ }^{36-38}$ We studied the influence of a double bond in two noncoordinating hydrophobic solvents, octadecene and octadecane. In ref. ${ }^{16}$, the authors observed no difference in Mn-doped $\mathrm{ZnO}$ NP shapes (tetrapods) when they used octadecane instead of octadecene. However, we see a dramatic difference in the NP shape evolution. The sample 
obtained in octadecane after 10 min (MZO-13) consists of solely pyramid-like NPs. No dimers or trimers obtained by tip-to-base attachment are observed. (Fig. S6, SI). The sample isolated after 60 min shows dimers, but with only minimal smoothing. These data clearly demonstrate that although the reaction mechanism is retained, i.e., pyramids assemble into nails due to oriented attachment, in octadecane this process is significantly decelerated. We hypothesize that a double bond of octadecene facilitates the oriented attachment. To the best of our knowledge, however, there is no discussion in literature about the influence of a solvent double bond on the oriented attachment as well as there are no examples of reactions in octadecane allowing for such an attachment. In eicosane with a higher boiling point, the oriented attachment of FeS NPs has been observed. ${ }^{39}$ Because octadecene and octadecane have similar boiling temperatures, the differences observed in our case are best attributed to the double bond influence. When the oriented attachment mechanism is not observed (tetrapod formation), ${ }^{16}$ the presence of a double bond makes no difference.

Along with the two non-coordinating solvents, we explored an influence of a coordinating solvent, benzyl ether, on the NP shape. ZnO NPs of various shapes were prepared in benzyl ether, including dendritic-like structures. ${ }^{40}$ In this solvent ether oxygen could bind to a metal, although because of steric hindrances from the two benzyl groups, this should be a weak interaction. Nevertheless, the MZO-15 NPs formed immediately after the ODA addition are different from the NPs obtained in octadecene: They are concave nanopyramids (chanterelle-like NPs) (Fig. S7a, SI), whose random assembly gives rise to branched, dendritic particles (Fig. S7b, SI, MZO-16) and eventually to larger aggregates (Fig. S7c, SI, MZO-17). Thus, benzyl ether changes the shape of the final product by changing the shape of the in-situ generated seeds and their attachment to each other. ${ }^{41}$ 


\section{Reaction without ODA}

$\mathrm{Zn}(\mathrm{St})_{2}$ is reported to be stable in solution up to $320{ }^{\circ} \mathrm{C}$ but decomposes immediately in the presence of ODA. ${ }^{26}$ To verify the stability of the mixture of $\mathrm{Zn}(\mathrm{St})_{2}$ and $\mathrm{Mn}(\mathrm{St})_{2}$ (with $10 \mathrm{~mol} . \%$ of the latter) in octadecene, the solution was heated at the reaction temperature for $100 \mathrm{~min}$. The TEM image of the product after the 100 min reaction (Fig. 4a, MZO-18) shows large NP dimers, consisting of concave triangular pyramids. Each pyramid looks like a short nail, with a nail head of about $29-31 \mathrm{~nm}$ in size and the total nail length of approximately the same size (Fig. 4a). The nail foot has a triangular cross-section (the red arrow), while a nail head shows a truncated triangular cross-section (the blue arrow) as can be seen from the image of a single particle. HRTEM and STEM dark-field images (Fig. 4b and c) clearly show connections of NPs in dimers via basal planes. A comparison of the STEM dark-field image and EDS maps (Fig. 4, d-f) demonstrates that the $\mathrm{Zn}, \mathrm{Mn}$, and O maps have the same shape as the NPs in the dark-field image, revealing that the all species are located in the same NPs. This is also confirmed by the good fit of the superposition of Zn, Mn, and O maps (Fig. 4g).
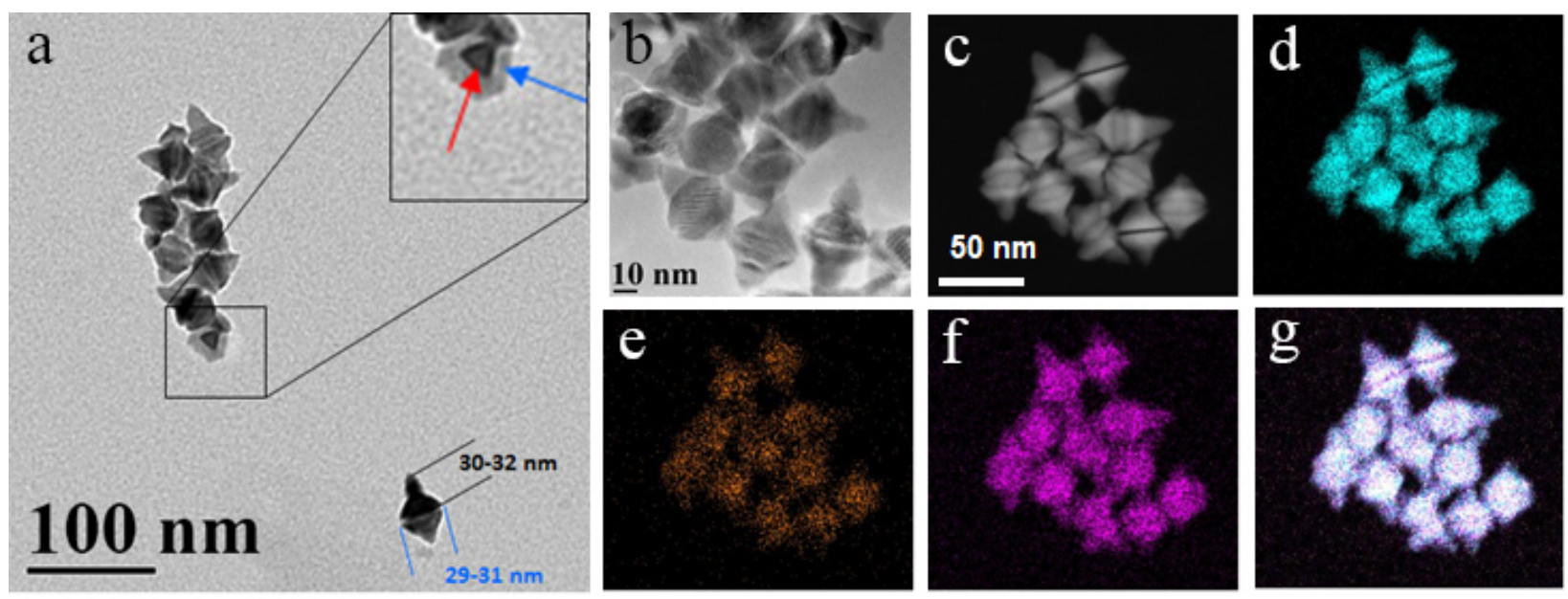

Figure 4. TEM (a), HRTEM (b), and dark-field STEM (c) images as well as STEM EDS maps of Zn (d), Mn (e), O (f), and their superposition (g) of MZO-18 NPs prepared without the ODA 
addition for $100 \mathrm{~min}$. The red arrow in the (a) inset shows a cross-section of the nail foot, while the blue arrow indicates the nail head. HRTEM clearly shows a connection of NPs in dimers.

It comes as no surprise that Mn-doped ZnO NPs can be stabilized without ODA, as stearate anions can serve as surfactants. However, because their content is low, much larger particles form compared to those prepared with ODA. At the same time, the question arises: How and when do these NPs start forming? We noticed that, after approximately 15-20 min, the solution started turning from colorless to yellow (a similar color change was observed upon addition of ODA) and becoming turbid. To follow the NP growth without ODA, we took probes after 20 and 40 min (timing started when the reaction temperature, $300{ }^{\circ} \mathrm{C}$, was reached) and also analyzed the final product after $100 \mathrm{~min}$ (Fig. S8, SI. MZO-19). Through the probes, we see progression from predominantly dimers of small, concave triangular pyramids (with some unreacted precursor on the grid background) to much larger particles of a similar shape. It is worth noting that the final NPs produced in the synthesis where probes were taken, are much larger than those formed in the undisturbed reaction. This outcome indicates that taking probes does disturb (accelerates) the particle growth but does not change the final NP shape. The other observation is that without ODA large concave pyramids favor the base-to-base attachment vs. tip-to-base oriented attachment seen in the ODA stabilized NPs. Similar NP dimers were observed for ZnO NPs synthesized in ODE, however, in the presence of ODA. ${ }^{26}$ The authors suggested that this phenomenon is due to welldeveloped facets at the basal plane. They also hypothesized that these pyramids might be polarized, with the dipole moment perpendicular to the basal plane, which would create two differently charged pyramids attracting each other, when the basal planes are terminated with either zinc or oxygen ions. ${ }^{26}$ Considering that nanopyramid pairs are also dominant in the Mn-doped ZnO NP samples prepared without ODA, we find this hypothesis compelling. 


\section{XPS/XRD/EDS characterization of Mn-doped ZnO NPs}

The XRD pattern of MZO-1 NPs prepared in ODE (for 10 min after the addition of solid ODA) is presented in Figure 5a. The pattern matches literature data for hexagonal wurtzite $\mathrm{ZnO}$ nanoparticles (JCPDS 36-1451) ${ }^{25}$ with a slight angle downshift relative to pure $\mathrm{ZnO}$ (Fig. S9, SI). This shift indicates the increased lattice constants of doped $\mathrm{ZnO} N \mathrm{Ns}^{25}$

The XRD patterns of all samples discussed in this paper are very similar, indicating that even for different NP shapes, the crystalline structure remains unchanged. For MZO-1, the mean value of size distribution derived from full pattern fitting (Fig. S10) and assuming an isotropic model is $93.8 \AA$ (average of long and short dimensions for elongated particles). ${ }^{42}$ However, the MZO-1 pattern clearly shows anisotropic peak broadening, in part due to anisotropic crystallite morphology, with full-width-at-half-maximum values between 0.35 and $1.2^{\circ}$ in $2 \theta$. In particular, the (002) reflection is very narrow compared to all other reflections. This finding is consistent with our observation of elongated crystallites using TEM.

The XPS spectra for the same samples were recorded to assess the oxidation state of metals, elemental composition, and distribution of species. The survey XPS spectrum of the sample discussed above shows $\mathrm{Zn}, \mathrm{Mn}, \mathrm{O}, \mathrm{C}$, and $\mathrm{Si}$ (Fig. 5b). The last is normally an impurity. The HR XPS Zn 2p spectrum (Fig. 5c) shows BE of $1021.3 \mathrm{eV}$, which is typical for $\mathrm{Zn}^{2+}{ }^{43}$ The position of the HR XPS Mn 2p3/2 peak (Fig. 5d) is at $640.8 \mathrm{eV}$, which is consistent with $\mathrm{Mn}^{2+}$ in $\mathrm{MnO}^{44-45}$ 

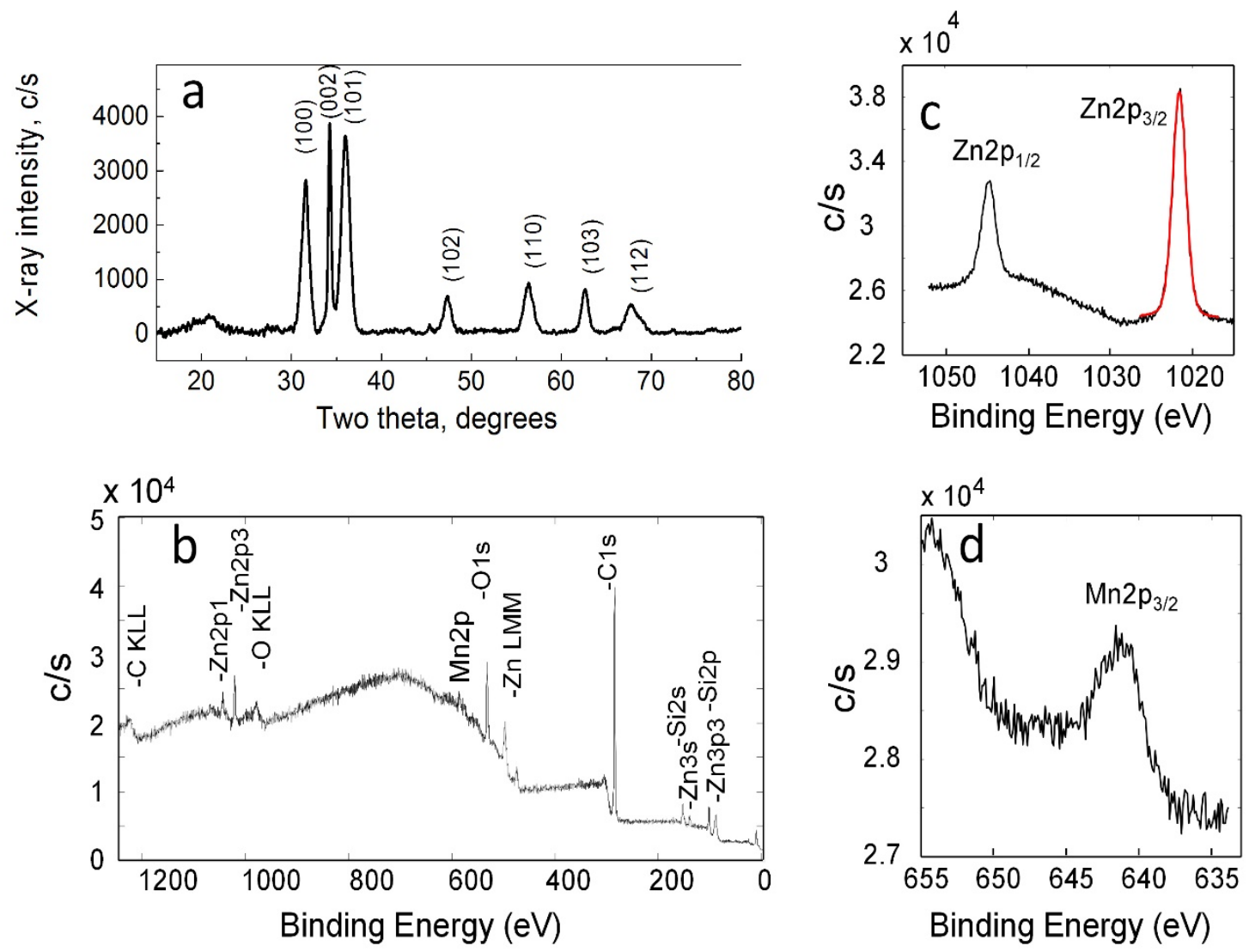

Figure 5. XRD pattern (a), survey XPS spectrum (b) and high resolution XPS Zn 2p (c) and Mn 2p $3 / 2(d)$ of MZO-1 NPs.

The elemental composition of the Mn-doped ZnO NPs was evaluated by EDS to obtain bulk values and by XPS for $2 p$ and $3 p$ electrons to obtain surface and undersurface contents. Because the kinetic energy of $3 p$ electrons is much higher than that of $2 p$ electrons, the former probe the deeper layers in the NP, while the latter assess metal contents on the NP surface. ${ }^{46-47}$ 
Table 2. EDS/XPS data and the band gaps calculated from the Tauc plots for representative Mndoped $\mathrm{ZnO}$ NP samples.

\begin{tabular}{|c|c|c|c|c|}
\hline \multirow[b]{2}{*}{ Sample notation } & \multicolumn{2}{|c|}{$\begin{array}{l}\text { Mn content, at. } \%^{\mathrm{a}} \\
\text { from XPS }\end{array}$} & \multirow[t]{2}{*}{$\begin{array}{l}\text { Mn, at. } \\
\% \text { b, from } \\
\text { EDS }\end{array}$} & \multirow[t]{2}{*}{$\begin{array}{l}\text { Band gap, } \\
\text { eV }\end{array}$} \\
\hline & $2 p$ & $3 p$ & & \\
\hline MZO-1 & 19.3 & 17.6 & 11.2 & 3.38 \\
\hline MZO-3 & 18.4 & 18.0 & 7.9 & 3.39 \\
\hline MZO-8 & 11.1 & 14.8 & 35.2 & 3.31 \\
\hline MZO-16 & 5.3 & 5.2 & 5.6 & 3.47 \\
\hline MZO-18 & 27.6 & 24.6 & 10.2 & 3.36 \\
\hline
\end{tabular}

${ }^{\mathrm{a}, \mathrm{b}} \mathrm{Mn}$ and $\mathrm{Zn}$ atomic contents constitute $100 \%$; neither oxygen nor carbon is taken into consideration.

Comparison of the EDS and XPS data shows that the surface of MZO-1, MZO-3, and MZO-18 synthesized in octadecene is enriched with Mn. For the MZO-8 sample isolated immediately after the ODA addition (with stopped stirring), the surface Mn content is 11.1 at.\%, while the bulk content is 35.2 at.\%, revealing that $\mathrm{Mn}$ stearate decomposes first, catalyzing the decomposition of $\mathrm{Zn}$ stearate (consistent with the data on the $\mathrm{Zn}(\mathrm{St})_{2}$ thermal stability ${ }^{26}$ ). The compositions of MZO1 and MZO-3 (both on the surface and bulk) are not identical, although quite close. In the case of MZO-18, i.e., dimers of concave pyramids prepared without ODA, the Mn content on the surface exceeds that of the bulk content by nearly a factor of three, indicating a significant surface enrichment with $\mathrm{Mn}$ and most likely a $\mathrm{ZnO}$ core. The Mn surface enrichment could be a result of recrystallization within NPs at the reaction temperature.

For the sample synthesized in benzyl ether, both surface and bulk contain only 5.3-5.6 at.\% of $\mathrm{Mn}$. We think that in this case, the $\mathrm{Mn}(\mathrm{St})_{2}$ decomposition is impeded compared to that of $\mathrm{Zn}(\mathrm{St})_{2}$ 
due to the better coordination of $\mathrm{Mn}(\mathrm{St})_{2}$ with the benzyl ether oxygen, leading to the low $\mathrm{Mn}$ incorporation.

\section{Optical properties}

Doping of $\mathrm{ZnO}$, a well-known wide band gap $(3.3 \mathrm{eV})$ semiconductor, was widely pursued to affect the band gap, and thus, semiconducting properties, which could be used in solar cells, luminescent devices, and chemical sensors. ${ }^{48-50}$ In this work, however, we explore the optical properties solely as an illustration of morphological or structural changes of Mn-doped ZnO NPs.

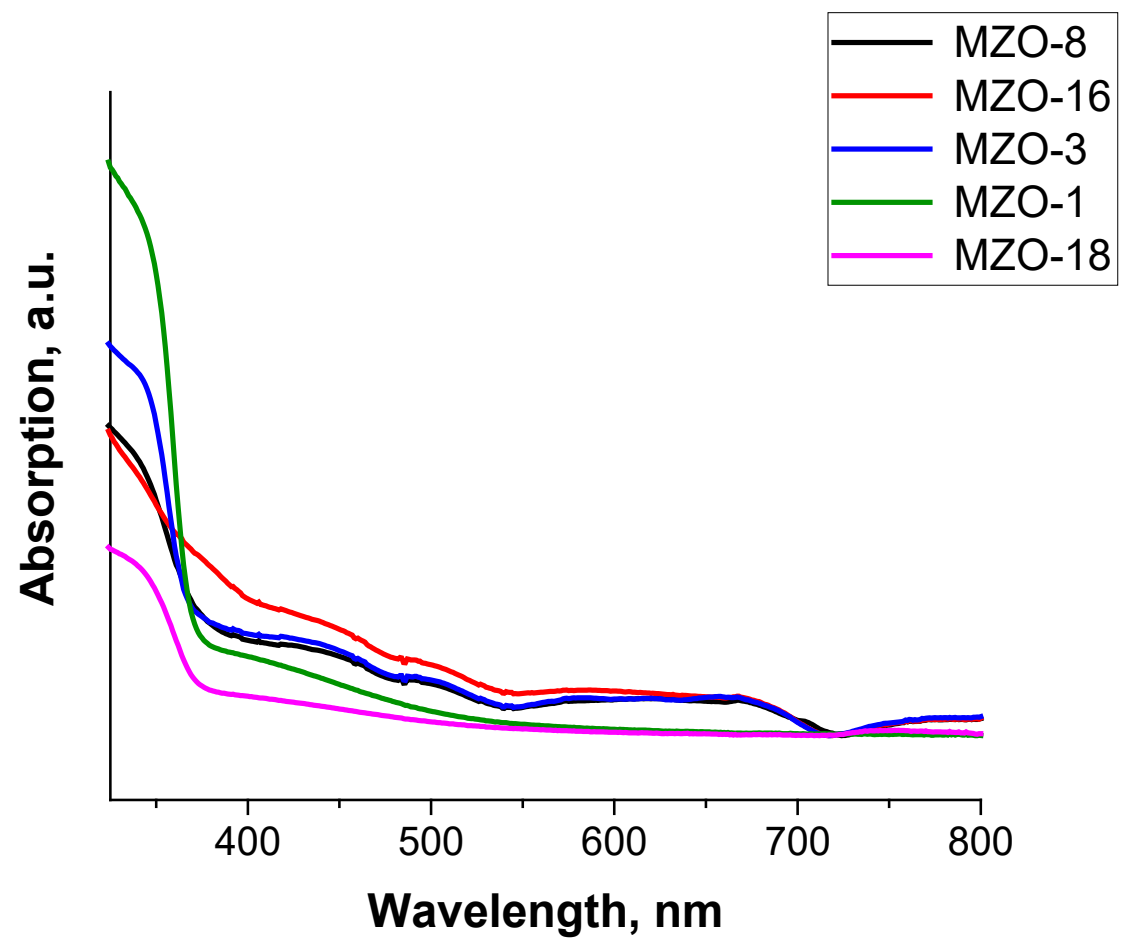

Figure 6. UV-Vis absorbance spectra of the key samples listed in Table 2.

Figure 6 displays UV-Vis absorbance spectra of the key samples listed in Table 2. For all Mndoped $\mathrm{ZnO}$ NPs, there are slight blue shifts (with respect to that of pure $\mathrm{ZnO}^{51}$ ) of the absorption edge near $360 \mathrm{~nm}$ corresponding to the main semiconducting transition. This is consistent with the well-established fact that $\mathrm{MnO}$ has a larger band gap (4.2 eV) compared to that of $\mathrm{ZnO}$ (3.3 $\mathrm{eV})$, leading to the shift of the absorption edge to shorter wavelengths. ${ }^{48,52}$ This is often explained 
by the energy band reconstruction of $\mathrm{ZnO}$ due to $\mathrm{Mn}$ doping. ${ }^{25}$ It is noteworthy, however, the opposite phenomenon, i.e., a shift to the longer wavelengths, was also reported ${ }^{53-55}$ and attributed to the strong exchange interactions between the $d$ electrons of Mn and the $s$ and $p$ electrons of the host band. The values of band gaps determined from the Tauc plots (an example is shown in Fig. S11, SI, for MZO-18) and presented in Table 2 may reflect contributions from both phenomena. On the other hand, there is no correlation between the band gap increase and an increase of the $\mathrm{Mn}$ content, Table 2), indicating the complexity of this phenomenon.

Similar to preceding reports, ${ }^{25,52,56}$ we observed a broad absorption band, the width of which is dependent on the sample. The lowest absorption in the range of 380-500 nm is observed for large concave pyramid dimers synthesized without ODA (MZO-18). The highest absorption in the range of 380-700 $\mathrm{nm}$ is detected for dendritic particles synthesized in benzyl ether (MZO-16). Very similar absorption in the visible region was found for nail-like NPs prepared with hot ODA addition (MZO-3) and for elongated structures formed by the tip-to-base assembly of several nanopyramids at the beginning of the reaction (MZO-8). Surprisingly, nail-like NPs prepared by addition of solid ODA (MZO-1) compared to those prepared by hot ODA addition (MZO-3) show lower absorption in the $380-500 \mathrm{~nm}$ range and none above $500 \mathrm{~nm}$, despite the NP shape and the Mn contents being very similar. A possible difference is in the Mn content distribution from the surface to the core. At the same time, the band gaps of MZO-1 and MZO-3 are nearly identical (3.38 and $3.39 \mathrm{eV}$, respectively), indicating similarity of these semiconducting structures. Because the sources of the absorption in the visible light region are considered controversial, ${ }^{25,57}$ we are limiting our discussion to obvious differences in NP morphologies, although it is quite likely that other factors are contributing to this phenomenon. As for band gaps, the highest band gap (3.47 
$\mathrm{eV})$ is obtained for MZO-16 with a dendritic shape, while the lowest band gap (3.31 eV), which is close to that of bulk $\mathrm{ZnO}(3.3 \mathrm{eV})$, is found for the self-assembled pyramids (MZO-8).

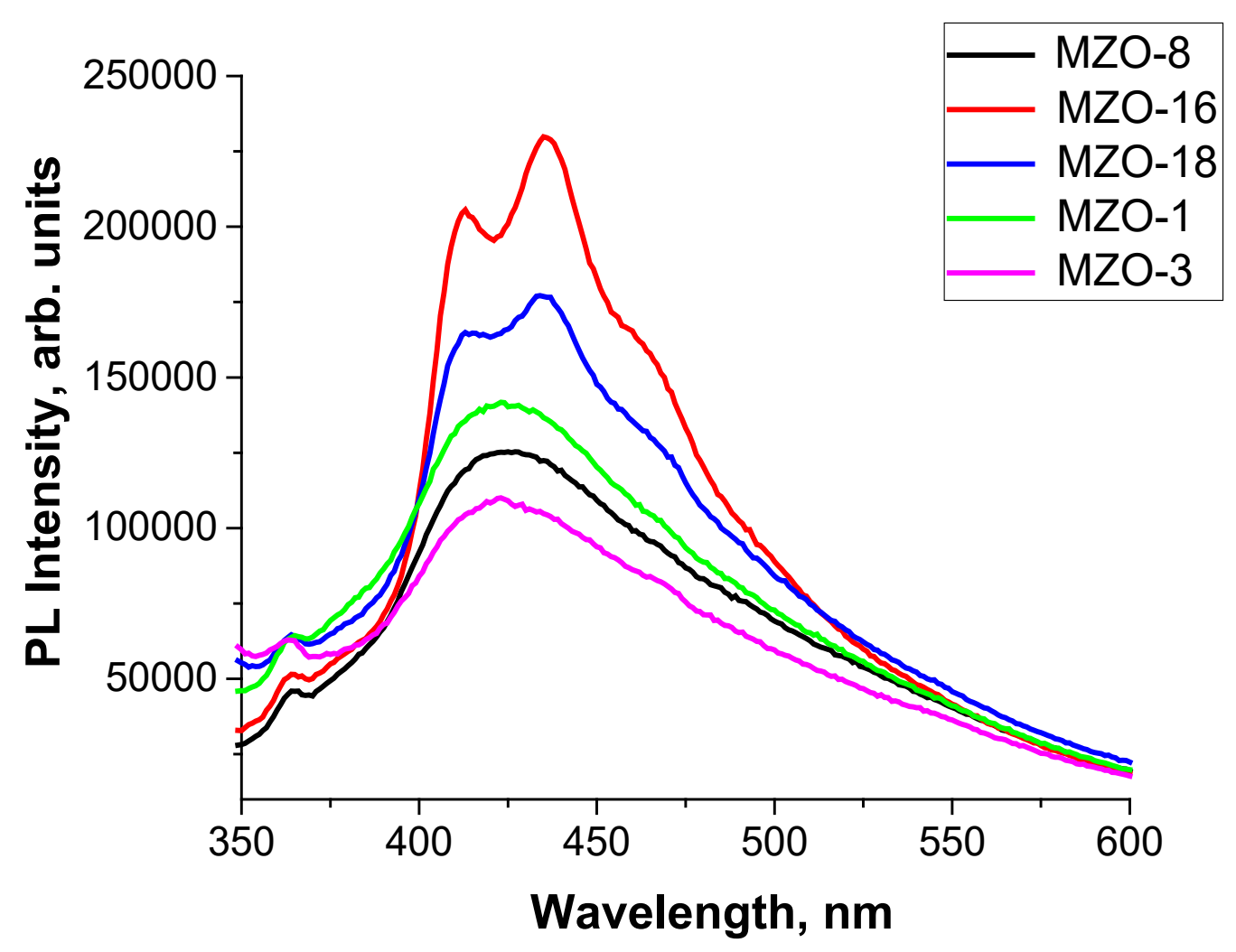

Figure 7. PL spectra of the key samples listed in Table 2.

Figure 7 presents PL spectra of the same key samples listed in Table 2. All the PL spectra show a weak peak at $\sim 370 \mathrm{~nm}$ which can be assigned to the near-band-gap emission ${ }^{48,58}$ and much stronger emission in the range of $400-500 \mathrm{~nm}$. At the same time, the emission at $520 \mathrm{~nm}$ attributed to surface traps ${ }^{48,59}$ is barely noticeable, indicating that the surface traps are quenched. It is worth noting that $\mathrm{ZnO}$ nanopyramids reported elsewhere ${ }^{26}$ do not show $\mathrm{PL}$ in the $400-500 \mathrm{~nm}$ range, unless they were etched with an acid. Undoped and doped ZnO NPs of different shapes were reported to demonstrate significant emission changes, depending on capping molecules and solvents used. ${ }^{16,60}$ The PL spectra of MZO-16 (dendrites) and MZO-18 (pyramid dimers) display 
three bands at $\sim 412,435$, and 464 (shoulder) nm, while the spectra of other samples show a broad peak with a maximum at $\sim 425 \mathrm{~nm}$. The presence of three emission bands in the $400-500 \mathrm{~nm}$ range shows that defect states are introduced at several discrete energies within the band gap. ${ }^{56,61}$ For elongated samples (MZO-1, MZO-3, and MZO-8), the defect states are partially quenched, which is reflected in featureless peaks with lower intensities. This allows us to conclude that PL properties are shaped dependent for the Mn-doped ZnO NPs discussed in this work.

\section{Conclusion}

We developed novel, nail-like Mn-doped ZnO NPs in non-coordinating solvents (octadecene, octadecane) and proposed a mechanism of their formation. According to this mechanism, initially, triangular pyramids are formed, which are prone to tip-to-base oriented attachment, accompanied by smoothing. This mechanism leads to single crystalline, nail-like NPs. The presence of a double bond in the solvent (octadecene vs. octadecane) promotes oriented attachment. In weakly coordinating solvent such as benzyl ether, initially Chanterelle-like NPs are formed, which assemble into irregularly shaped, dendritic structures, revealing once more the importance of the solvent in determining the NP shape. When the NP formation is carried out without ODA, which serves both as surfactant and reaction initiator, it occurs very slowly and leads to large, concave pyramids that predominantly assemble into dimers via their basal planes.

The UV-Vis and PL spectra data are dependent on morphology and structure of the NPs, reaffirming the utility of the shape and composition control to modify electrooptical properties.

\section{ASSOCIATED CONTENT}


Supporting Information. TEM, HRTEM, XRD, and UV-Vis data. This material is available free of charge via the Internet at http://pubs.acs.org.

AUTHOR INFORMATION

Corresponding Author

*lybronst@indiana.edu

\section{Author Contributions}

The manuscript was written through contributions of all authors.

\section{Present Addresses}

$\dagger$ Department of Chemical and Biomolecular Engineering, University of Houston, Houston TX 77204

\section{ACKNOWLEDGMENT}

This work was made possible due to the support of the Indiana University Department of Chemistry. We also thank the Indiana University Nanoscale Characterization Facility for access to the instrumentation as well as NSF grant \#CHE-1048613 which funded the Empyrean from PANalytical.

\section{References}

1. Watson, P. A., Function follows form: generation of intracellular signals by cell deformation. FASEB Journal 1991, 5, 2013-2019. 
2. Fanger, C. M.; Zweifach, A.; Dolmetsch, R. E.; Hoth, M.; Lewis, R. S., Function follows form. The role of store-operated calcium channels in T-cell activation. Cell. Physiol. Biochem. 1997, 7, 203-218.

3. Tait, S. L., Function Follows Form: Exploring Two-Dimensional Supramolecular Assembly at Surfaces. ACS Nano 2008, 2, 617-621.

4. $\quad$ Szarko, J. M.; Guo, J.-C.; Liang, Y.-Y.; Lee, B.-D.; Rolczynski, B. S.; Strzalka, J.; Xu, T.; Loser, S.; Marks, T. J.; Yu, L.-P.; Chen, L.-X., When function follows form: effects of donor copolymer side chains on film morphology and BHJ solar cell performance. Adv. Mater. 2010, 22, $5468-5472$.

5. Laskar, M.; Skrabalak, S. E., A balancing act: manipulating reactivity of shape-controlled metal nanocatalysts through bimetallic architecture. J. Mater. Chem. A 2016, 4, 6911-6918.

6. Biacchi, A. J.; Schaak, R. E., Ligand-Induced Fate of Embryonic Species in the ShapeControlled Synthesis of Rhodium Nanoparticles. ACS Nano 2015, 9, 1707-1720.

7. Popczun, E. J.; Roske, C. W.; Read, C. G.; Crompton, J. C.; McEnaney, J. M.; Callejas, J. F.; Lewis, N. S.; Schaak, R. E., Highly branched cobalt phosphide nanostructures for hydrogenevolution electrocatalysis. J. Mater. Chem. A 2015, 3, 5420-5425.

8. Amiens, C.; Chaudret, B.; Ciuculescu-Pradines, D.; Colliere, V.; Fajerwerg, K.; Fau, P.; Kahn, M.; Maisonnat, A.; Soulantica, K.; Philippot, K., Organometallic approach for the synthesis of nanostructures. New J. Chem. 2013, 37, 3374-3401. 
9. Pu, Y.-J.; Morishita, N.; Chiba, T.; Ohisa, S.; Igarashi, M.; Masuhara, A.; Kido, J., Efficient Electron Injection by Size- and Shape-Controlled Zinc Oxide Nanoparticles in Organic LightEmitting Devices. ACS Appl. Mater. Interfaces 2015, 7, 25373-25377.

10. Yadavalli, T.; Shukla, D., Role of metal and metal oxide nanoparticles as diagnostic and therapeutic tools for highly prevalent viral infections. Nanomedicine (N. Y., NY, U. S.) 2017, 13, 219-230.

11. Lee, I.; Albiter, M. A.; Zhang, Q.; Ge, J.; Yin, Y.; Zaera, F., New nanostructured heterogeneous catalysts with increased selectivity and stability. Phys. Chem. Chem. Phys. 2011, $13,2449-2456$.

12. Perry, J. L.; Herlihy, K. P.; Napier, M. E.; DeSimone, J. M., PRINT: A Novel Platform Toward Shape and Size Specific Nanoparticle Theranostics. Acc. Chem. Res. 2011, 44, 990-998.

13. Regulacio, M. D.; Han, M.-Y., Multinary I-III-VI2 and I2-II-IV-VI4 Semiconductor Nanostructures for Photocatalytic Applications. Acc. Chem. Res. 2016, 49, 511-519.

14. Fukuzumi, S.; Yamada, Y., Shape- and Size-Controlled Nanomaterials for Artificial Photosynthesis. ChemSusChem 2013, 6, 1834-1847.

15. Cho, S.; Jung, S.-H.; Lee, K.-H., Morphology-Controlled Growth of ZnO Nanostructures Using Microwave Irradiation: from Basic to Complex Structures. J. Phys. Chem. C 2008, 112, 12769-12776.

16. Yang, Y.; Jin, Y.; He, H.; Wang, Q.; Tu, Y.; Lu, H.; Ye, Z., Dopant-Induced Shape Evolution of Colloidal Nanocrystals: The Case of Zinc Oxide. J. Am. Chem. Soc. 2010, 132, 13381-13394. 
17. Ayudhya, S. K. N.; Tonto, P.; Mekasuwandumrong, O.; Pavarajarn, V.; Praserthdam, P., Solvothermal Synthesis of $\mathrm{ZnO}$ with Various Aspect Ratios Using Organic Solvents. Cryst. Growth Des. 2006, 6, 2446-2450.

18. Khazaei, M.; Hosseini, M. S., Synthesis hydrophilic hybrid nanoparticles and its application in wettability alteration of oil-wet carbonate rock reservoir. Pet. Sci. Technol. 2017, Ahead of Print.

19. El-Nahhal, I. M.; Salem, J. K.; Tabasi, N. S.; Hempelmann, R.; Kodeh, F. S., Synthesis and structural characterization of $\mathrm{ZnO}$-and $\mathrm{CuO}-\mathrm{NPs}$ supported mesoporous silica materials (hexagonal SBA-15 and lamellar-SiO2). Chem. Phys. Lett. 2018, 691, 211-218.

20. Wesselinowa, J. M.; Apostolov, A. T., A possibility to obtain room temperature ferromagnetism by transition metal doping of $\mathrm{ZnO}$ nanoparticles. J. Appl. Phys. 2010, 107, 053917/1-053917/5.

21. Bandyopadhyay, P.; Dey, A. B., Ruma; ; Nandy, P.; Das, S., Effect of Cu doping in ZnO nanoparticles for increased voltage generation, storage capacity, and energy conversion efficiency in photoelectrochemical cell. Energy Sourc. A 2016, 38, 1833-1839.

22. Kant, S.; Kumar, A., A comparative analysis of structural, optical and photocatalytic properties of $\mathrm{ZnO}$ and $\mathrm{Ni}$ doped $\mathrm{ZnO}$ nanospheres prepared by sol-gel method. Adv. Mater. Lett. 2012, 3, 350-354.

23. Clavel, G.; Willinger, M.-G.; Zitoun, D.; Pinna, N., Solvent dependent shape and magnetic properties of doped $\mathrm{ZnO}$ nanostructures. Adv. Funct. Mater. 2007, 17, 3159-3169. 
24. Bilecka, I.; Luo, L.; Djerdj, I.; Rossell, M. D.; Jagodic, M.; Jaglicic, Z.; Masubuchi, Y.; Kikkawa, S.; Niederberger, M., Microwave-Assisted Nonaqueous Sol-Gel Chemistry for Highly Concentrated ZnO-Based Magnetic Semiconductor Nanocrystals. J. Phys. Chem. C 2011, 115, 1484-1495.

25. Yang, Y.; Li, Y.; Zhu, L.; He, H.; Hu, L.; Huang, J.; Hu, F.; He, B.; Ye, Z., Shape control of colloidal $\mathrm{Mn}$ doped $\mathrm{ZnO}$ nanocrystals and their visible light photocatalytic properties. Nanoscale 2013, 5, 10461-10471.

26. Chen, Y.; Kim, M.; Lian, G.; Johnson, M. B.; Peng, X., Side Reactions in Controlling the Quality, Yield, and Stability of High Quality Colloidal Nanocrystals. J. Am. Chem. Soc. 2005, 127, 13331-13337.

27. Ibarra-Sanchez, J. J.; Fuentes-Ramirez, R.; Roca, A. G.; del Puerto Morales, M.; CabreraLara, L. I., Key Parameters for Scaling up the Synthesis of Magnetite Nanoparticles in Organic Media: Stirring Rate and Growth Kinetic. Ind. Eng. Chem. Res. 2013, 52, 17841-17847.

28. Bai, Y.; Yang, T.; Gu, Q.; Cheng, G.; Zheng, R., Shape Control Mechanism of Cuprous Oxide Nanoparticles in Aqueous Colloidal Solutions. Powder Technol. 2012, 227, 35-42.

29. Pourrahimi, A. M.; Liu, D.; Andersson, R. L.; Stroem, V.; Gedde, U. W.; Olsson, R. T., Aqueous Synthesis of (210) Oxygen-Terminated Defect-Free Hierarchical ZnO Particles and Their Heat Treatment for Enhanced Reactivity. Langmuir 2016, 34, 11002-11013.

30. Dai, Y.; Cobley, C. M.; Zeng, J.; Sun, Y.; Xia, Y., Synthesis of Anatase TiO2 Nanocrystals with Exposed $\{001\}$ Facets. Nano Lett. 2009, 9, 2455-2459. 
31. Ludi, B.; Sueess, M. J.; Werner, I. A.; Niederberger, M., Mechanistic aspects of molecular formation and crystallization of zinc oxide nanoparticles in benzyl alcohol. Nanoscale 2012, 4, 1982-1995.

32. Niederberger, M.; Coelfen, H., Oriented Attachment and Mesocrystals: Non-classical Crystallization Mechanisms Based on Nanoparticle Assembly. Phys. Chem. Chem. Phys. 2006, 8, 3271-3287.

33. Ataee-Esfahani, H.; Skrabalak, S. E., Attachment-based growth: building architecturally defined metal nanocolloids particle by particle. $R S C A d v . \mathbf{2 0 1 5}, 5,47718-47727$.

34. Narayanaswamy, A.; Xu, H.; Pradhan, N.; Kim, M.; Peng, X., Formation of Nearly Monodisperse In2O3 Nanodots and Oriented-Attached Nanoflowers: Hydrolysis and Alcoholysis vs Pyrolysis. J. Am. Chem. Soc. 2006, 128, 10310-10319.

35. Tan, S. T.; Umar, A. A.; Balouch, A.; Yahaya, M.; Yap, C. C.; Salleh, M. M.; Oyama, M., $\mathrm{ZnO}$ nanocubes with (101) basal plane photocatalyst prepared via a low-frequency ultrasonic assisted hydrolysis process. Ultrason. Sonochem. 2014, 21, 754-760.

36. Krishnapriya, R.; Praneetha, S.; Vadivel Murugan, A., Energy-efficient, microwaveassisted hydro/solvothermal synthesis of hierarchical flowers and rice grain-like $\mathrm{ZnO}$ nanocrystals as photoanodes for high performance dye-sensitized solar cells. CrystEngComm 2015, 17, 83538367.

37. Saric, A.; Stefanic, G.; Drazic, G.; Gotic, M., Solvothermal Synthesis of Zinc Oxide Microspheres. J. Alloys Compd. 2015, 652, 91-99. 
38. Raula, M.; Biswas, M.; Mandal, T. K., Ionic Liquid-based Solvent-induced Shape-tunable Small-sized ZnO Nanostructures with Interesting Optical Properties and Photocatalytic Activities. RSC Advances 2014, 4, 5055-5064.

39. Lai, H.-Y.; Chen, C.-J., Shape-controlled synthesis of iron sulfide nanostructures via oriented attachment mechanism. J. Cryst. Growth 2009, 311, 4698-4703.

40. Liu, X.; Swihart, M. T., A General Single-pot Heating Method for Morphology, Size and Luminescence-controllable Synthesis of Colloidal ZnO Nanocrystals. Nanoscale 2013, 5, 80298036.

41. Weiner, R. G.; Kunz, M. R.; Skrabalak, S. E., Seeding a New Kind of Garden: Synthesis of Architecturally Defined Multimetallic Nanostructures by Seed-Mediated Co-Reduction. Acc. Chem. Res. 2015, 48, 2688-2695.

42. Popa, N. C.; Teodorescu, C. M.; Frunza, S., New analytical approximation of diffraction size broadened peak profile for spherical crystallites with a lognormal distribution. J. Appl. Crystallogr. 2010, 43, 1027-1030.

43. Bera, S.; Prince, A. A. M.; Velmurugan, S.; Raghavan, P. S.; Gopalan, R.; Panneerselvam, G.; Narasimhan, S. V., Formation of Zinc Ferrite by Solid-state Reaction and its Characterization by XRD and XPS. J. Mater. Sci. 2001, 36, 5379-5384.

44. Xu, G.; Zhang, L.; Guo, C.; Gu, L.; Wang, X.; Han, P.; Zhang, K.; Zhang, C.; Cui, G., Manganese Monoxide/Titanium Nitride Composite as High Performance Anode Material for Rechargeable Li-ion Batteries. Electrochim. Acta 2012, 85, 345-351. 
45. Debnath, B.; Salunke, H. G.; Shivaprasad, S. M.; Bhattacharyya, S., Surfactant-Mediated Resistance to Surface Oxidation in MnO Nanostructures. ACS Omega 2017, 2, 3028-3035.

46. Somorjai, G. A., In Chemistry in Two Dimensions: Surfaces, Cornell University Press: Ithaca, NY, 1981, p 41.

47. Powell, C. J.; Jablonski, A., Evaluation of Calculated and Measured Electron Inelastic Mean Free Paths Near Solid Surfaces. J. Phys. Chem. Ref. Data 1999, 28, 19.

48. Viswanatha, R.; Sapra, S.; Sen Gupta, S.; Satpati, B.; Satyam, P. V.; Dev, B. N.; Sarma, D. D., Synthesis and Characterization of Mn-Doped ZnO Nanocrystals. J. Phys. Chem. B 2004, $108,6303-6310$.

49. Singh, A. K.; Thool, G. S.; Bangal, P. R.; Madhavendra, S. S.; Singh, S. P., Low Temperature Mn Doped ZnO Nanorod Array: Synthesis and Its Photoluminescence Behavior. Ind. Eng. Chem. Res. 2014, 53, 9383-9390.

50. Santra, P. K.; Kamat, P. V., Mn-Doped Quantum Dot Sensitized Solar Cells: A Strategy to Boost Efficiency over 5\%. J. Am. Chem. Soc. 2012, 134, 2508-2511.

51. Djerdj, I.; Garnweitner, G.; Arcon, D.; Pregelj, M.; Jaglicic, Z.; Niederberger, M., Diluted magnetic semiconductors: $\mathrm{Mn} / \mathrm{Co}$-doped $\mathrm{ZnO}$ nanorods as case study. J. Mater. Chem. 2008, 18, $5208-5217$.

52. Wang, Y. S.; Thomas, P. J.; O'Brien, P., Optical Properties of ZnO Nanocrystals Doped with Cd, Mg, Mn, and Fe Ions. J. Phys. Chem. B 2006, 110, 21412-21415.

53. Guo, Y.; Cao, X.; Lan, X.; Zhao, C.; Xue, X.; Song, Y., Solution-Based Doping of Manganese into Colloidal ZnO Nanorods. J. Phys. Chem. C 2008, 112, 8832-8838. 
54. Bylsma, R. B.; Becker, W. M.; Kossut, J.; Debska, U.; Yoder-Short, D., Dependence of energy gap on $\mathrm{x}$ and $\mathrm{T}$ in zinc manganese selenide (Zn1-xMnxSe): the role of exchange interaction. Phys. Rev. B: Condens. Matter 1986, 33, 8207-15.

55. Zhang, H.-W.; Shi, E.-W.; Chen, Z.-Z.; Liu, X.-C.; Xiao, B.; Song, L.-X., Optical and magnetic resonance investigations of $\mathrm{Zn1-xMnxO}$ magnetic semiconductors. J. Magn. Magn. Mater. 2006, 305, 377-380.

56. Inamdar, D. Y.; Pathak, A. K.; Dubenko, I.; Ali, N.; Mahamuni, S., Room Temperature Ferromagnetism and Photoluminescence of Fe Doped ZnO Nanocrystals. J. Phys. Chem. C 2011, 115, 23671-23676.

57. Johnson, C. A.; Kittilstved, K. R.; Kaspar, T. C.; Droubay, T. C.; Chambers, S. A.; Mackay Salley, G.; Gamelin, D. R., Mid-gap electronic states in Zn1-xMnxO. Phys. Rev. B: Condens. Matter Mater. Phys. 2010, 82, 115202/1-115202/11.

58. Li, J.; Fan, H.; Chen, X.; Cao, Z., Structural and photoluminescence of Mn-doped ZnO single-crystalline nanorods grown via solvothermal method. Colloids Surf., A 2009, 349, 202-206.

59. Guo, L.; Yang, S.; Yang, C.; Yu, P.; Wang, J.; Ge, W.; Wong, G. K. L., Synthesis and Characterization of Poly(vinylpyrrolidone)-Modified Zinc Oxide Nanoparticles. Chem. Mater. 2000, 12, 2268-2274.

60. Liu, X.; Swihart, M. T., A general single-pot heating method for morphology, size and luminescence-controllable synthesis of colloidal $\mathrm{ZnO}$ nanocrystals. Nanoscale 2013, 5, 80298036. 
61. Ponnusamy, R.; Selvaraj, S. C.; Ramachandran, M.; Murugan, P.; Nambissan, P. M. G.; Sivasubramanian, D., Diverse Spectroscopic Studies and First-Principles Investigations of the Zinc Vacancy Mediated Ferromagnetism in Mn-Doped ZnO Nanoparticles. Cryst. Growth Des. 2016, 16, 3656-3668. 
TOC graphic

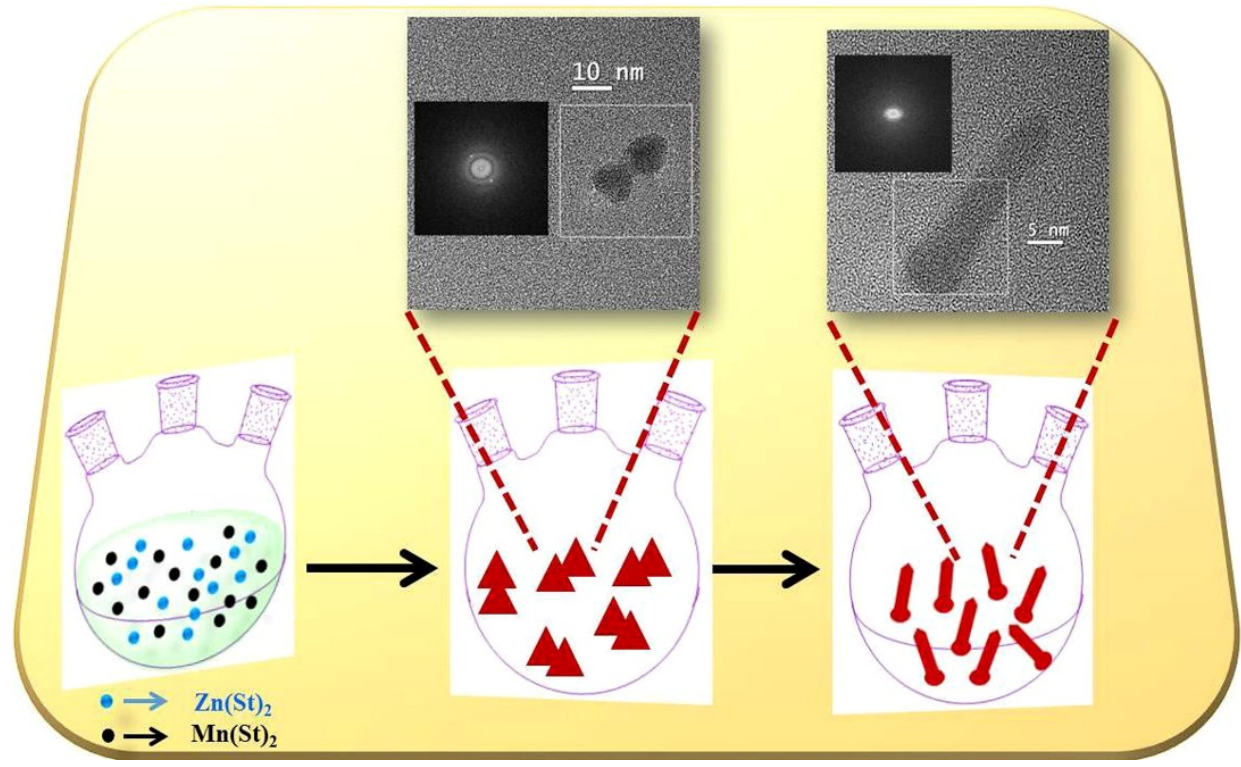

